\title{
A STUDY ON BIPEDAL AND MOBILE ROBOT BEHAVIOR THROUGH MODELING AND SIMULATION
}

\author{
Nirmala \\ Department of Mechatronics \\ Faculty of Engineering \\ Swiss German University \\ Tangerang 15339, Indonesia \\ Email: nirmala_91@hotmail.com
}

\author{
Prianggada I Tanaya \\ Department of Industrial Engineering \\ Faculty of Engineering \\ Swiss German University \\ Tangerang 15339, Indonesia \\ Email: prianggada.itanaya@sgu.ac.id
}

\author{
Maralo Sinaga \\ Department of Mechatronics \\ Faculty of Engineering \\ Swiss German University \\ Tangerang 15339, Indonesia \\ Email: maralo.sinaga@iuli.ac.id
}

\begin{abstract}
Bipedal robot-mobile robot in general-is important for many purposes in various fields including engineering field. For this robot type, it is important to understand the robot dynamic characteristics. The characteristics are walking behavior, exploration behavior, human-following behavior, aversive behavior, and robot stability. This study focuses to understand these characteristics by means of Virtual Robot Experimentation Platform (V-REP). The results are also compared to those results obtained from the actual experiments of literatures. The results are of the following. The walking behavior strongly depends on the height and size of the robot step. On the exploration behavior, increasing the wheel mass reduces the variance in the traveled distance but increases the deviation on the path. On the human-following behavior, the faster the person walks, the harder the robot follows. The aversive behavior strongly depends on the ability of the motor to generate acceleration. The robot reaches its maximum stability with the location of the center of mass at $30.0 \mathrm{~mm}$. Finally, the differences between the simulation results and those of experiments are relatively small.
\end{abstract}

Keywords: Bipedal Robot; Mobile Robot; Robot Behavior; Robot Modeling; Robot Simulation

\section{INTRODUCTION}

Robots were originally used to perform single and simple tasks such as pick-and-place activity. Nowadays, the developed robots are so advanced that they can perform difficult and complex tasks [1-11].

Many aspects of robots have been studied independently. Reference [12] studied the biologically motivated push strategies for $3 \mathrm{D}$ bipedal robot. Reference [13] studied the trajectory of joint space for the optimal hip-mass of the robot. Reference [14] studied the robot characteristics in performing walking, rolling,

Received: March 22, 2015; received in revised form: April 6, 2015; accepted: May 14, 2015; available online: June 17, 2015. and standing. Reference [15] studied the aspect of adaptive control stability. However, there are lack of coherent assessments on the various dynamic characteristics of the mobile robots.

Building and developing a robot to fulfill a specific function require careful design and analysis. In the preliminary stages, it is necessary to build a prototype in order to evaluate the design. On these phases, simulation is capable to provide a virtual robot prototype, which can be used to study and analyze the robot behavior [16].

The principles for robot modeling and simulation have been advised by Ref. [17]. The sample framework has been proposed by Ref. [18]. This framework demonstrates the use of the simulation to model and analyze mobile robot behavior. In particular, the software called Virtual Robot Experimentation Platform (V-REP) is capable to simulate the robots of types: bipedal robot, human-follower transporter robot, and Autonomous Guided Vehicle (AGV) robot. In general, the robot simulation process follows these steps depicted in Fig. 1.

This study focuses on the following dynamic characteristics of the mobile robots: walking behavior, exploration behavior, human-following behavior, aversive behavior, and robot stability.

\section{METHODS}

The robot dynamic characteristics will be studied via a simulation software. The robot and its obstacles are created within Virtual Robotic Experimentation Platform (V-REP). Firstly, the robot mechanical structure is produced with a computer-aided design (CAD) software. Secondly, we specify the robot dynamic 
Cite this article as: Nirmala, P. I. Tanaya, and M. Sinaga, "A study on bipedal and mobile robot behavior through modeling and simulation," CommIT Journal, vol. 9, no. 1, pp. 1-10, 2015.

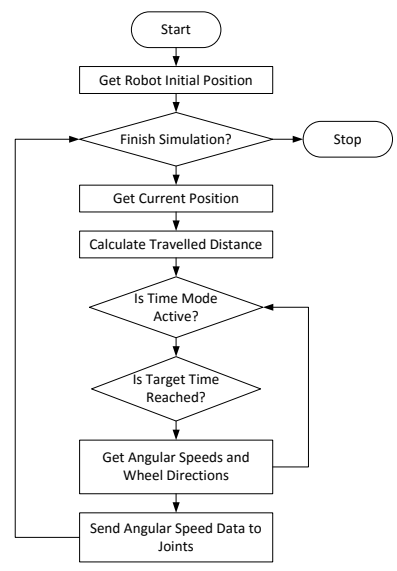

Fig. 4. Explorational Algorithm Flowchart.

circle denotes the person and the prism denotes the robot. The prism vertex is the robot head direction. The distances $x_{1}$ and $y_{1}$ are the relative position of the person with respect to the robot. $D$ is the distance between the centers of the two objects.

The algorithm of the human following behavior begins by acquiring the values of $x_{1}, y_{1}$, and $\beta$. The distance $D$ is calculated by

$$
D=\sqrt{x_{1}^{2}+x_{1}^{2}} .
$$

In addition, the corresponding angle $\alpha$ are calculated by:

$$
\alpha=\tan ^{-1}\left|\frac{x_{1}}{y_{1}}\right|
$$

for $y_{1}$ negative. However, when $y_{1}$ is positive, the formulas is

$$
\alpha=\pi-\tan ^{-1}\left|\frac{x_{1}}{y_{1}}\right| .
$$

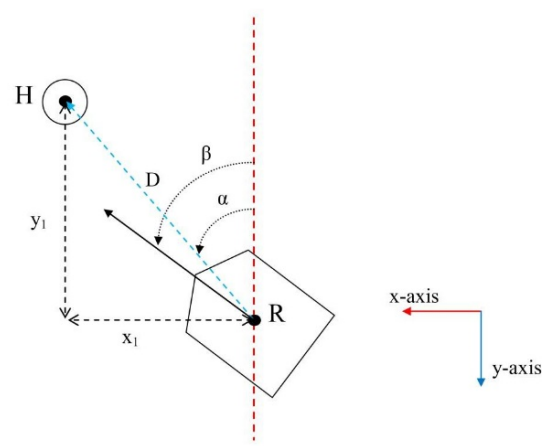

Fig. 5. Human and Transporter_MT position.
If $\alpha$ and $\beta$ are on the same side of the reference line, then the rotation angle to be performed by the robot is the absolute difference of the two angles $\alpha$ and $\beta$. Depending on $\alpha$ and $\beta$, the robot will either rotate the left or to the right.

Another feasible configuration is for $\alpha$ and $\beta$ at different side of the reference line. The algorithm will use the absolute values of $\alpha$ and $\beta$ and will add the two. If the resulting angle $\gamma$ is greater than $2 \pi$, then the rotation angle to be performed will be $2 \pi-\gamma$. The rotation direction depends only on $x_{1}, \alpha$, and $\beta$.

When the initial target position $A$ is obtained, the robot rotates itself and heads towards the target $A$. The robot wheels will move oppositely with the robot centerline as the pivot. The robot rotating speed $\omega$ is calculated by

$$
\omega=\frac{v_{l}-v_{r}}{d / 2},
$$

where $v_{l}$ and $v_{r}$ are the tangential speeds of the left and right wheel of the robot. $d$ denotes the distance between the wheels.

When the robot has reached the desired orientation, the robot approaches the target at a constant speed while maintaining its relative distance beyond a threshold. The threshold $r$ is user-defined and should be provided by considering the robot geometry. Once the robot entered the threshold, the robot will acquire the new target position $B$, which is the new position of the person.

The aversive behavior of AGV_TP will utilize the VREP functions to acquire the relative and absolute positions with respect to interacting objects. The behavior is derived on the basis of the interaction depicted in Fig. 6. In the figure, the green box represents AGV_TP which possesses the aversive behavior. The red box symbolizes the moving obstacles.

AGV_TP moves in a specified direction at the speed $v_{1}$. The moving obstacle moves towards AGV_TP from the left at the speed of $v_{2}$. AGV_TP and the obstacle is separated by the distances of $x_{1}$ and $y_{1}$ in the $x$ - and $y$-directions, respectively. The distances $x_{2}, y_{2}$, and

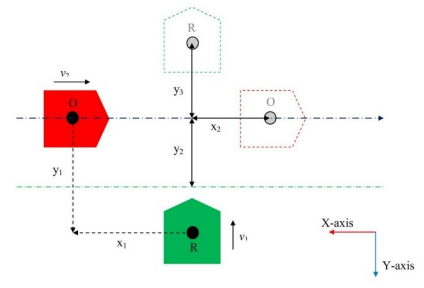

Fig. 6. Position Layout of Aversive Behavior. 
Cite this article as: Nirmala, P. I. Tanaya, and M. Sinaga, "A study on bipedal and mobile robot behavior through modeling and simulation,” CommIT Journal, vol. 9, no. 1, pp. 1-10, 2015.

$y_{3}$ denote the thresholds that will be used to decide whether the robot has safe distance to move forward without colliding the obstacle. These thresholds, as well as the speeds $v_{1}$ and $v_{2}$ are user-defined.

At the beginning of the simulation, the obstacle and robot are set to move at a constant velocity. Then, the robot will evaluate whether it has reached the critical region. The variables $t_{1}$ and $t_{2}$ denote the time for the robot and obstacle to pass the critical region and they can be calculated by

$$
\begin{aligned}
& t_{1}=\frac{x_{1}-x_{2}}{v_{1}} \\
& t_{2}=\frac{y_{1}-y_{2}}{v_{2}} .
\end{aligned}
$$

If $t_{1}$ is greater than or equal to $t_{2}$, the robot will collide with the obstacle. Thus, the robot has to wait the obstacle passes the region. If $t_{1}$ is smaller $t_{2}$, the robot should have time to pass the critical region without colliding the obstacle and the robot should maintain its speed [29].

\section{RESULTS AND DISCUSSION}

\section{A. Simulation based Analysis}

Figure 7 shows the result of the stability testing by adjusting the center of mass (COM) coordinates of the hip. The time taken for the robot to tilt by an angle of $30^{\circ}$ is measured for each case. The hip mass is kept constant at $0.5 \mathrm{~kg}$ and variation is made on the relative $y$-ordinate only. The robot is most stable when the relative $y$-ordinate of COM is $30.0 \mathrm{~mm}$ in which the time taken to tilt by $30^{\circ}$ is $45.35 \mathrm{~s}$.

The stability test result of BR-4 is shown at Table I. The hip mass is varied as well as the relative coordinate of $y z$-plane of the COM of the hip. Similarly the time taken for the robot to tilt by an angle of $30^{\circ}$ is measured in each case. The robot is most stable when the relative coordinates of $y z$-plane of the hip's COM

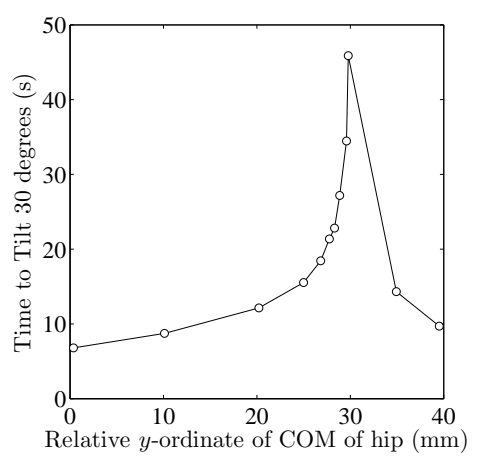

Fig. 7. The Stability Test of the BR_SS Robot.

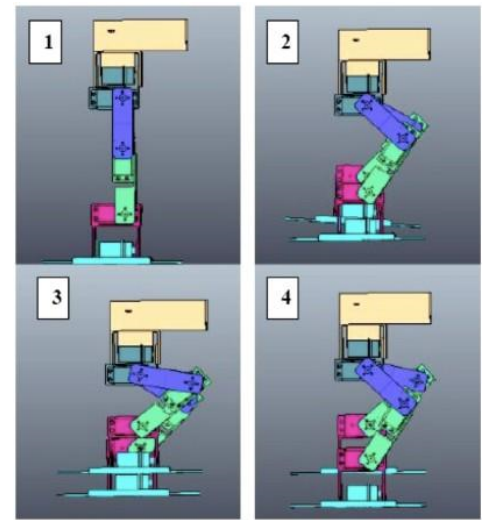

Fig. 8. Walking Gait of BR_SS Robot When the Robot Hip is Maintained Statically.

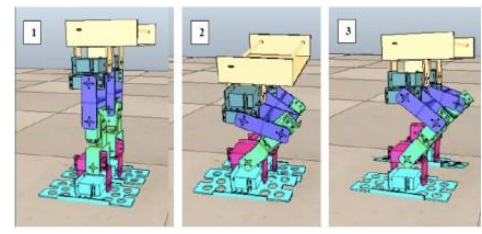

Fig. 9. Walking Gait of BR_SS Robot When the Robot Hip is Moving.

are $-20.0 \mathrm{~mm}$ and $0.0 \mathrm{~mm}$ respectively. The time taken to tilt on that configuration is $13.00 \mathrm{~s}$.

On the issue of the walking behavior of the bipedal robots, the simulation results are shown in Figs. 8 and 9. The former figure shows that the robot walks by cycling the legs alternatively up and down with the hip in a static condition. The latter figure shows the case where the hip is not maintained statically.

To execute walking motion into BR-4 robot, an inverse kinematics (IK) module is used for each leg. The left leg is supposed to move first followed by the right leg. However, with this approach, the robot jumps

TABLE I

STABILITY TEST RESUlt of BR-4.

\begin{tabular}{cccc}
\hline $\begin{array}{c}\text { Hip Mass } \\
(\mathrm{kg})\end{array}$ & $\begin{array}{c}\text { Rel. } y \text {-ord. } \\
(\mathrm{mm})\end{array}$ & $\begin{array}{c}\text { Rel. } z \text {-ord. } \\
(\mathrm{mm})\end{array}$ & $\begin{array}{c}\text { Time to Tilt } 30^{\circ} \\
(\mathrm{s})\end{array}$ \\
\hline 0.3 & 0.0 & 0.0 & 5.50 \\
0.3 & -10.0 & 0.0 & 7.15 \\
0.3 & -20.0 & 0.0 & 13.00 \\
0.3 & -10.0 & 10.0 & 6.95 \\
0.3 & -10.0 & 20.0 & 6.80 \\
0.5 & 0.0 & 0.0 & 3.65 \\
0.5 & -10.0 & 0.0 & 5.25 \\
0.5 & -20.0 & 0.0 & 5.10 \\
0.5 & -10.0 & 10.0 & 5.10 \\
0.5 & -10.0 & 20.0 & 5.00 \\
\hline
\end{tabular}

Rel. = Relative, ord. $=$ ordinate 
Cite this article as: Nirmala, P. I. Tanaya, and M. Sinaga, "A study on bipedal and mobile robot behavior through modeling and simulation," CommIT Journal, vol. 9, no. 1, pp. 1-10, 2015.

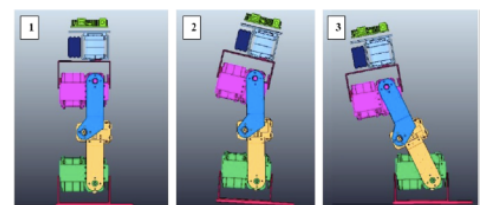

Fig. 10. BR-4 Jumping Instead of Walking.

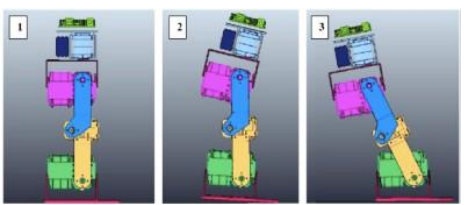

Fig. 11. The BR-4 Robot Executes the Left Leg Only.

instead of walks around as shown in Fig. 10.

During the second testing of the robot, a delay is introduced between the execution of the left leg and the right leg (see Fig. 11). However, the robot executes only the left leg movement without moving the right leg; hence, the robot loses its stability and fell. The problem persists when the IK calculation is performed continuously for each leg and at the same time, the robot is expected to walk with the alternative legs.

The robot exploration behavior is measured by the variation of the left and right wheel speeds, the coefficient of friction between the wheels and the floor, and the caster wheel's mass. The experiments are performed with the assumption that the motor can introduce the high level of acceleration. The simulation time is fixed at $5 \mathrm{~s}$. The first experiment is conducted by keeping the velocities of the wheels constant at 20 RPM and the coefficient of friction at 1.0. The caster wheel's mass is varied to see how it affects the robot straight-line motion. The result are plotted in Figs. 12 and 13. Those results show that the highest variations in the distance and the deviation occur at the mass of $0.7 \mathrm{~kg}$. In general, increasing the mass reduces the traveled distance but increases the variation. This phenomenon is likely due to the fact that the caster wheel's inertia is greater as the mass increases.

In the next experiment, the caster wheel's mass and the velocities are fixed constant at $0.1 \mathrm{~kg}$ and 20 RPM, respectively, but the coefficient of friction between the wheels and the floor is varied. The results are shown in Fig. 14 and 15. This experiment indicates that the traveled distance decreases with increasing friction. On the other hand, the deviation fluctuates between $5 \mathrm{~mm}$ and $40 \mathrm{~mm}$ when the friction coefficient increases gradually from 0.2 to 1.0 .

For the AGV robot, the experiment is carried out by maintaining the platform's mass constant while varying

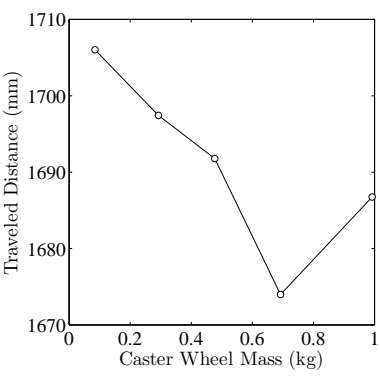

Fig. 12. The Variation of the Traveled Distance with the Caster Wheel's Mass for the Transporter Robot.

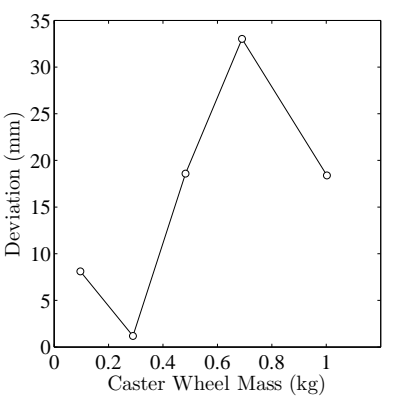

Fig. 13. The Variation of the Traveled Distance with the Friction Coefficient for the Transporter Robot.

the pulley's mass. During the experiment, the curve motion of $\mathrm{AGV}$ _TP is observed and the diameter of the circle path made by the robot is measured. The measured data are then compared to the calculated diameter value. As illustrated in Fig. 16, the diameter of the circle for different velocity $v_{1}$ and $v_{2}$ is given as:

$$
\begin{aligned}
\frac{x}{x+w} & =\frac{v_{1}}{v_{2}}, \\
x & =w \frac{v_{1}}{v_{2}-v_{1}}, \\
D_{\text {curve }} & =x+\frac{w}{2} .
\end{aligned}
$$

The errors are calculated and tabulated along with the measured values. Figure 17 shows the graph of the error variation based on the changing value of pulley mass.

From Fig. 17, it can be seen that the smaller masses of pulley $(2-6 \mathrm{~kg})$ resulted in relatively small errors compared to the larger ones $(8-10 \mathrm{~kg})$. Figure 18 shows the path of AGV_TP of the given pulley's mass. The yellow line is the path made with pulley's mass of $2 \mathrm{~kg}$ while the red line is the path made with pulley's mass of $10 \mathrm{~kg}$. The large inertia of the pulley resists the motor torque and as a result, the robot is unable 
Cite this article as: Nirmala, P. I. Tanaya, and M. Sinaga, "A study on bipedal and mobile robot behavior through modeling and simulation,” CommIT Journal, vol. 9, no. 1, pp. 1-10, 2015.

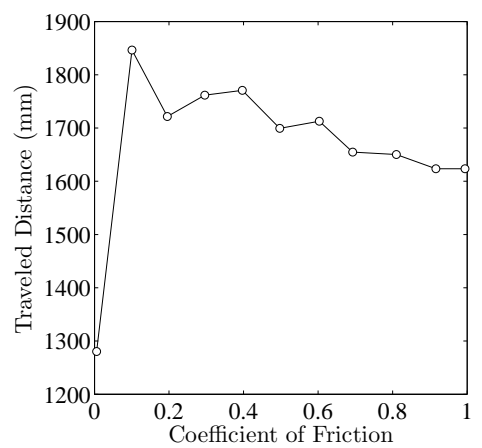

Fig. 14. Variation Of Deviation Versus Friction Coefficient (Transporter Robot).

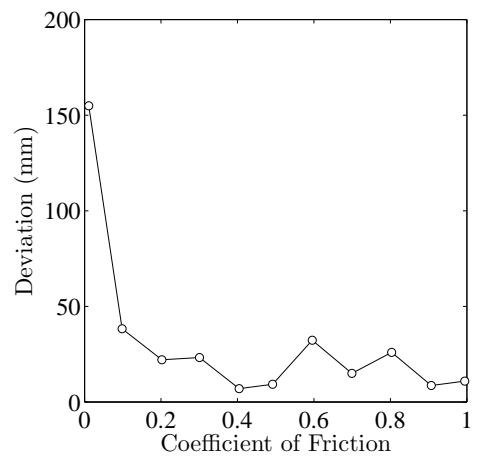

Fig. 15. Variation of the Deviation Versus the Coefficient of Friction of the Transporter Robot.

to perform the desired circular motion perfectly as specified through the path planner.

Another simulation experiment is performed by varying platform's mass instead of pulley's mass. Fig. 19 shows influence of varying platform's mass to path error. The largest error is made when the platform mass is around $80 \mathrm{~kg}$. It can be deduced that larger error are made with higher velocities, due to larger inertia of the platform which resists the motor torque. Two user-defined paths are drawn and a human model from V-REP library is employed to follow the path. Then, the transporter deploys a human-following algorithm to follow the person. To follow the person, two path shapes are prepared. The first path shape is like an eight number. There are several parameters which are varied in order to see the robot path execution. These are the person walking speed, the speed at which the robot rotates, the speed at which the robot approaches the person, the threshold distance between the person and the robot, and the initial positions of the robot. The results of the experiments are shown on Fig. 20.

The robot ran off its track because the human walk speed is very slow as shown in Fig. 20A. As a result,

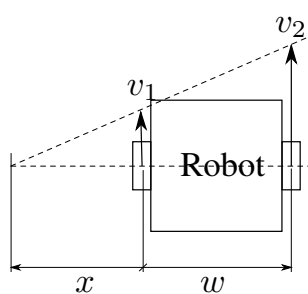

Fig. 16. The Curve Radius for Different Wheel Velocities.

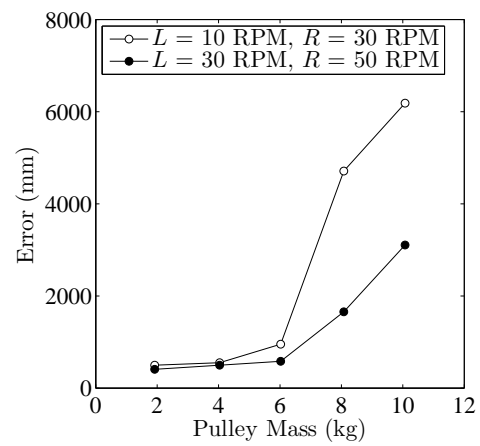

Fig. 17. Errors Versus Pulley Mass.

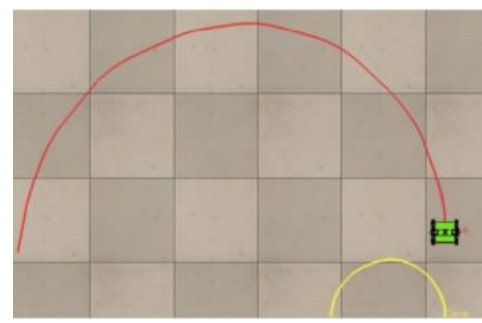

Fig. 18. Robot Circular Path of Different Pulley's Masses.

the robot collided with the person and bounced off the track.

Figure 20B shows the smoothest result among all tracked human paths. This may be caused by the threshold distance which does not allow the robot to come too close and thus not the slightest collision response is generated. It can be deduced that the for a good path pattern, a slow walking speed of the human is necessary but it has to be accompanied with large threshold distance so that no crash occurs. Figure 20D suggests that the human walk speed is very fast in comparison to the robot's execution. Hence, the robot's path is broken and not smooth. Towards the end of the track, the robot is unable to approach the person last position and it went beyond the track.

The second path shape is a sinusoidal wave. Different initial configurations are made and the results are shown at Fig. 21. It shows the pattern of robot following human at the front.

The robot aversive or protective behavior is studied 
Cite this article as: Nirmala, P. I. Tanaya, and M. Sinaga, "A study on bipedal and mobile robot behavior through modeling and simulation," CommIT Journal, vol. 9, no. 1, pp. 1-10, 2015.

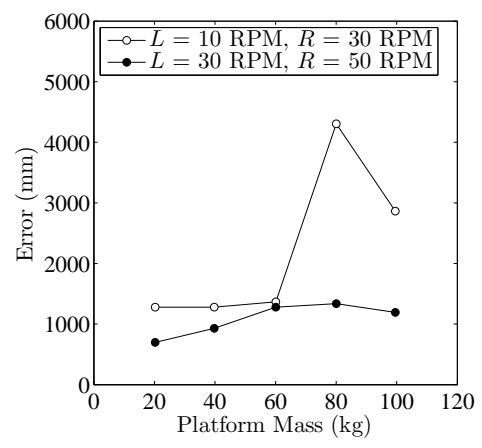

Fig. 19. Path Error Due to Varying Mass of the Platform.

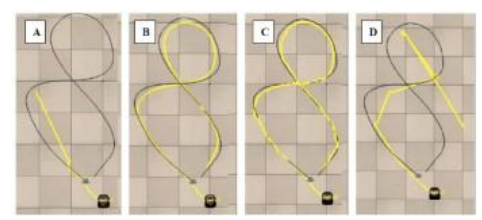

Fig. 20. Robot Following Human Behavior Darker Curve Shows Human Path from A-D.
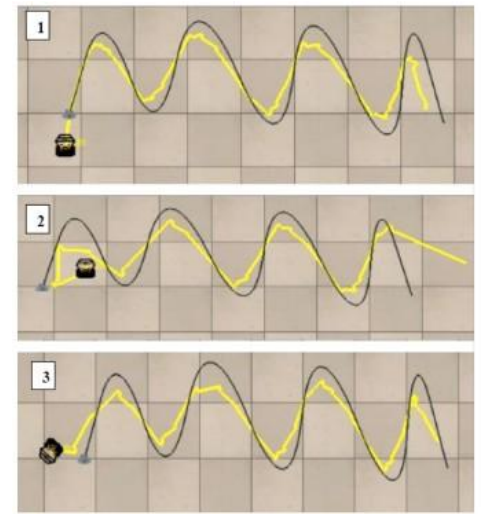

Fig. 21. Sinusoidal Following Path Pattern with Different Robot Setting.

by adjusting three parameters: the detection distance $y_{2}$ (see Fig. 6), the accelerations of the motors, and the initial configurations of the robot and obstacle. The robot velocity $v_{1}$ and obstacle velocity $v_{2}$ are maintained constant at $1.0 \mathrm{~m} / \mathrm{s}$. The initial configurations are shown in Fig. 22. The results of this case are summarized in Table II. The data show that the robot aversive behavior strongly depends on the acceleration of the motors and the robot initial configuration.

\section{B. Validation with Real Robot}

The walking behavior of the bipedal robot is compared to the actual robot with the second model of the simulation. The comparison is focused on the movement of the robot hip and is made qualitatively. In

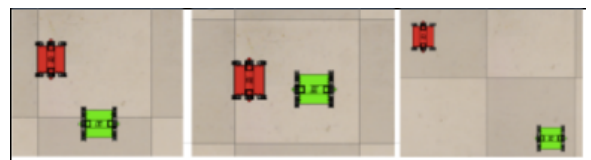

Fig. 22. The Three Initial Configurations of the AGV TP Robot for Studying the Aversive Behavior.

TABLE II

The Results of the RoBot Aversive Behavior EXPERIMENTS.

\begin{tabular}{cccc}
\hline Configuration & $\begin{array}{c}\text { Acceleration } \\
\left(\mathrm{mm} / \mathrm{s}^{2}\right)\end{array}$ & $\begin{array}{c}\text { Detection Distance } \\
(\mathrm{mm})\end{array}$ & Status \\
\hline 1 & 87.3 & 800 & Success \\
2 & 87.3 & 800 & Fail \\
3 & 87.3 & 800 & Fail \\
3 & 87.3 & 2000 & Success \\
3 & 174.5 & 800 & Fail \\
3 & 174.5 & 2000 & Success \\
2 & $>174.5$ & 800 & Fail \\
\hline
\end{tabular}

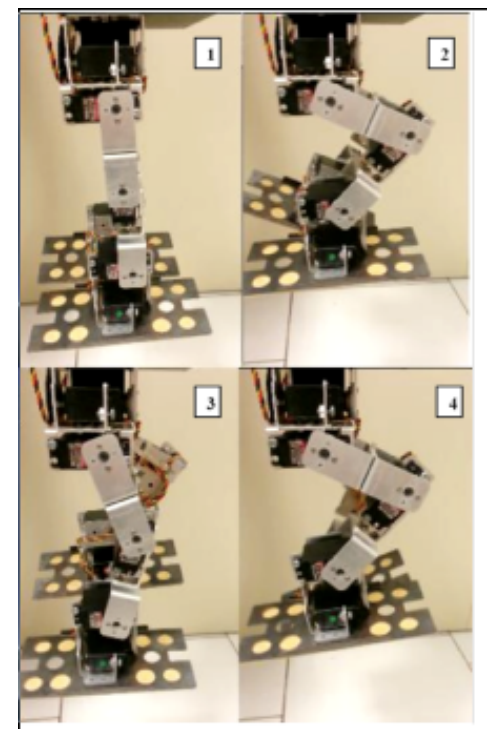

Fig. 23. Walking Gait of Physical Bipedal Robot on the Condition of Fixed Hip of BR_SS Robot [21, 22].

the case of the actual robot, the robot hip is maintained on a fixed position by means of cables. The result is shown in Fig. 23. The simulated robot response has previously shown in Fig. 8. By comparing the two figures, it suggests that the walking behavior are rather similar.

The simulated robot model is also compared the actual robot for the exploration behavior. In this case, the both robots are compared in their ability to move across a straight line. The results are shown in Figs. 24 and 25. Those figures indicate that the traveled distances 
Cite this article as: Nirmala, P. I. Tanaya, and M. Sinaga, "A study on bipedal and mobile robot behavior through modeling and simulation,” CommIT Journal, vol. 9, no. 1, pp. 1-10, 2015.

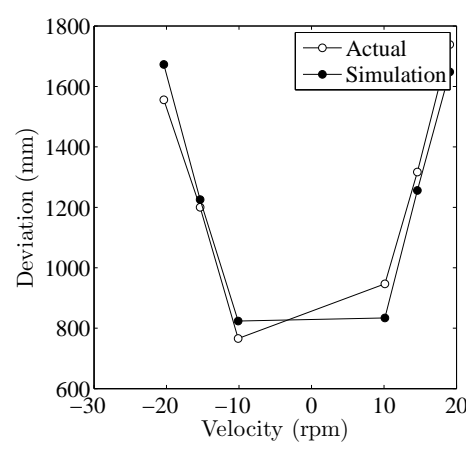

Fig. 24. A Comparison of the Traveled Distance of the Actual Robot and the Simulated Robot for Transporter_MT robot.

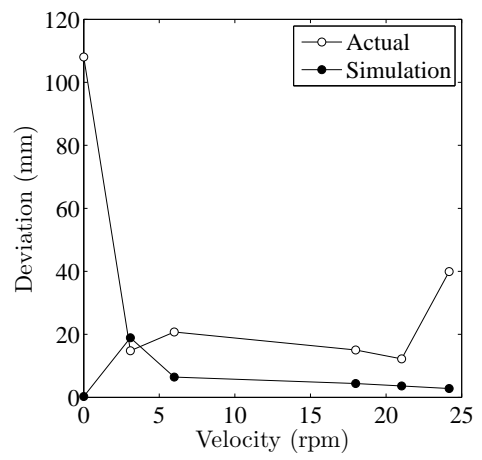

Fig. 25. A Comparison of the Deviations of the Actual Robot and the Simulated Robot for Transporter_MT Robot.

by the two robots are about the same in trend and in magnitude. However, the actual robot movement has slightly larger variation. The actual robot movement is clearly affected by the roughness of the floor, which changes the direction of the caster wheel easily.

As clearly shown in Table III, the actual robot deviates more the actual trajectory with a magnitude larger than that of the simulated robot. The simulated robot operates in an ideal condition where the left and right pulleys are identical and the driving motors are synchronized precisely. However, for the case of the actual robot, the pulleys are clearly not identical and the motors are not precisely synchronized.

The human-following behavior has been evaluated in Ref. [26] and their results are compared to the current simulation results. This comparison is shown in Fig. 26. In the actual robot, the robot trace is produced using a marker that placed the center of the robot platform. The marker leaves the robot trace on the lab floor where the experiment is performed. The results in Fig. 26 conclude that the two robots behave similarly.
TABLE III

Deviation of the Actual Robot and Simulated AGV_tP Robot on Motion along a Straight Line.

\begin{tabular}{ccc}
\hline Traveled Distance & \multicolumn{2}{c}{ Deviation } \\
\cline { 2 - 3 } & $\begin{array}{c}\text { Actual Robot } \\
(\mathrm{mm})\end{array}$ & $\begin{array}{c}\text { Simulated Model } \\
(\mathrm{mm})\end{array}$ \\
\hline 1000 & 10.0 & 4.8 \\
2000 & 15.0 & 10.1 \\
3000 & 25.0 & 12.8 \\
\hline
\end{tabular}

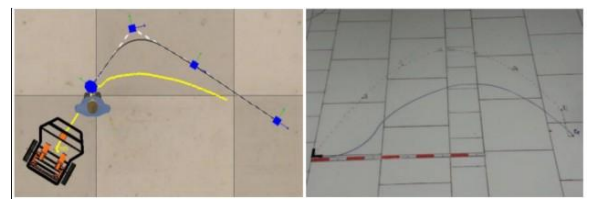

Fig. 26. Robot Path Validation between the Simulated Model and the Actual Robot for the Case of Transporter_MT Robot.

\section{CONCLUSIONS}

The behavior study of the bipedal and mobile robot are necessary for a preliminary study and can be done through simulation. The mechanical model with joint and link connection should be properly arranged to function similar to that of the real physical robot configuration. The modeling and simulation application tools such as V-REP are crucial. The robot behavior is affected by its own properties, the given task and its surrounding environment. There are small discrepancy in the robot responses between the actual and the simulated robots. This gap is important and can be used as information for configuring the robot control system.

\section{REFERENCES}

[1] A. Ames, "Human-inspired control of bipedal walking robots," Automatic Control, IEEE Transactions on, vol. 59, no. 5, pp. 1115-1130, May 2014.

[2] M. Al Lawati and C. Nielsen, "Hybrid virtual holonomic constraints for a 2-d.o.f. bipedal robot," in Electrical and Computer Engineering (CCECE), 2015 IEEE 28th Canadian Conference on, May 2015, pp. 320-325.

[3] A. Kumar, A. Krishnan, A. Sridhar, N. Kiruthika, and N. Prakash, "Design and fabrication of bipedal robot," in Computing, Communication and Networking Technologies (ICCCNT), 2014 International Conference on, July 2014, pp. 16.

[4] C. Chevallereau, J. Grizzle, and C.-L. Shih, "Asymptotically stable walking of a five-link un- 
Cite this article as: Nirmala, P. I. Tanaya, and M. Sinaga, "A study on bipedal and mobile robot behavior through modeling and simulation," CommIT Journal, vol. 9, no. 1, pp. 1-10, 2015.

deractuated 3-d bipedal robot," Robotics, IEEE Transactions on, vol. 25, no. 1, pp. 37-50, Feb 2009.

[5] C.-L. Shih, J. Grizzle, and C. Chevallereau, "Asymptotically stable walking of a simple underactuated 3d bipedal robot," in Industrial Electronics Society, 2007. IECON 2007. 33rd Annual Conference of the IEEE, Nov 2007, pp. 27662771.

[6] J. Pratt and G. Pratt, "Intuitive control of a planar bipedal walking robot," in Robotics and Automation, 1998. Proceedings. 1998 IEEE International Conference on, vol. 3, May 1998, pp. 2014-2021 vol.3.

[7] Y. Tsusaka and Y. Ota, "Wire-driven bipedal robot," in Intelligent Robots and Systems, 2006 IEEE/RSJ International Conference on, Oct 2006, pp. 3958-3963.

[8] A. Albert, M. Suppa, and W. Gerth, "Detection of stair dimensions for the path planning of a bipedal robot," in Advanced Intelligent Mechatronics, 2001. Proceedings. 2001 IEEE/ASME International Conference on, vol. 2, 2001, pp. 1291-1296 vol.2.

[9] H. Urahama, Y. Tazaki, and T. Suzuki, "Supervisory control of periodic stepping motion of a bipedal robot," in SICE Annual Conference (SICE), 2011 Proceedings of, Sept 2011, pp. 589594.

[10] C. Zaoui, O. Bruneau, F. Ouezdou, and A. Maalej, "Dynamic balance of a bipedal robot with trunk and arms subjected to 3d external disturbances," in Intelligent Robots and Systems, 2007. IROS 2007. IEEE/RSJ International Conference on, Oct 2007, pp. 4053-4058.

[11] T. Shimizu, S. Nakaura, and M. Sampei, "The control of a bipedal running robot based on output zeroing considered rotation of the ankle joint," in Decision and Control, 2006 45th IEEE Conference on, Dec 2006, pp. 6456-6461.

[12] J. Zhao, S. Schutz, and K. Berns, "Biologically motivated push recovery strategies for a $3 \mathrm{~d}$ bipedal robot walking in complex environments," in Robotics and Biomimetics (ROBIO), 2013 IEEE International Conference on, Dec 2013, pp. 1258-1263.

[13] N. Mir-Nasiri and H. Jo, "Joint space legs trajectory planning for optimal hip-mass carry walk of 4-dof parallelogram bipedal robot," in Mechatronics and Automation (ICMA), 2010 International Conference on, Aug 2010, pp. 616-621.

[14] M. Inaba, F. Kanehiro, S. Kagami, and H. Inoue,
"Two-armed bipedal robot that can walk, roll over and stand up," in Intelligent Robots and Systems 95. 'Human Robot Interaction and Cooperative Robots', Proceedings. 1995 IEEE/RSJ International Conference on, vol. 3, Aug 1995, pp. 297302 vol.3.

[15] J. Hu, J. Pratt, and G. Pratt, "Stable adaptive control of a bipedal walking; robot with cmac neural networks," in Robotics and Automation, 1999. Proceedings. 1999 IEEE International Conference on, vol. 2, 1999, pp. 1050-1056 vol.2.

[16] Q. Shujie, C. Lumin, W. Xinjie, and C. Rentao, "The design of bionic leg and motion simulation on passive dynamic bipedal robot," in Digital Manufacturing and Automation (ICDMA), 2010 International Conference on, vol. 2, Dec 2010, pp. 656-659.

[17] G. Pelz, Mechatronics Systems: Modelling and Simulation with HDLs. John Wiley \& Sons, 2003.

[18] D. Berenson, P. Abbeel, and K. Goldberg, A Robot Path Planning Framework that Learns from Experience. Berkeley, US: x, 2012.

[19] U. Nehmzow, "On the role of qualitative descriptions of behavior in mobile robotics research," in Proceedings of the 7th RoboCup Symposium, Padua, Italy, 2003.

[20] F. Ali, A. Amran, and A. Kawamura, "Bipedal robot walking strategy on inclined surfaces using position and orientation based inverse kinematics algorithm," in Control Automation Robotics Vision (ICARCV), 2010 11th International Conference on, Dec 2010, pp. 181-186.

[21] G. W. Setiono, "Kinematic model analysis and development of walking algorithm for 5 degree of freedom bipedal robot," Master's thesis, Department of Mechatronics, Faculty of Engineering, Swiss German University, 2012.

[22] A. F. Sutanto, "Design and implementation of fuzzy auto-balance control for bipedal robot," Master's thesis, Department of Mechatronics, Faculty of Engineering, Swiss German University, 2012.

[23] K. Marcellino, "Designing, developing and analyzing of maneuver walking gait for bipedal robot," Master's thesis, Bachelor Thesis, Department of Mechatronics, Faculty of Engineering, Swiss German University, 2013.

[24] S. Citra, "Development of linear and curve trajectory path planning for bipedal robot using camera vision," Master's thesis, Department of Mechatronics, Faculty of Engineering, Swiss German 
Cite this article as: Nirmala, P. I. Tanaya, and M. Sinaga, "A study on bipedal and mobile robot behavior through modeling and simulation,” CommIT Journal, vol. 9, no. 1, pp. 1-10, 2015.

University, 2013.

[25] F. K. Mista, "Development of trajectory planner based on lagrange polynomial and b-spline equations for an autonomous human follower transporter robot," Master's thesis, Department of Mechatronics, Faculty of Engineering, Swiss German University, 2013.

[26] W. Tjiu, "Development of execution and monitoring architecture for an autonomous human follower transporter robot," Master's thesis, Department of Mechatronics, Faculty of Engineering, Swiss German University, 2013.

[27] W. Tewira, "Development of path planning based on lagrange's polynomial method for dynamic obstacle avoidance of take over and cross road maneuver using kinect sensor of a mobile robot,"
Master's thesis, Department of Mechatronics, Faculty of Engineering, Swiss German University, 2013.

[28] E. Permadi, "Design and development of a mobile robot based on planning execution and monitoring architecture with integration of fusion sensor," Master's thesis, Department of Mechatronics, Faculty of Engineering, Swiss German University, 2013.

[29] N. Kita, F. Kanehiro, M. Morisawa, and K. Kaneko, "Obstacle detection for a bipedal walking robot by a fisheye stereo," in System Integration (SII), 2013 IEEE/SICE International Symposium on, Dec 2013, pp. 119-125. 